\title{
Caracterización ecológica de la macrofauna edáfica en dos sitios de bosque siempreverde en EI Salón, Sierra del Rosario, Cuba
}

\author{
Ecological characterization of soil macrofauna in two \\ evergreen forest sites at El Salón, Sierra del Rosario, Cuba
}

\author{
Grisel de la C Cabrera Dávila ${ }^{a *}$, Germán M López Iborra ${ }^{b}$ \\ *Autor de coprrespondencia: a Instituto de Ecología y Sistemática, Ministerio de Ciencia, Tecnología y Medio Ambiente, \\ Carretera de Varona 11835, Boyeros, C.P. 11900, La Habana 19, Cuba, tel. (537) 7643-8266, grisel17@ecologia.cu \\ ${ }^{\mathrm{b}}$ Universidad de Alicante, Departamento de Ecología/IMEM Ramón Margalef, Alicante, España.
}

\begin{abstract}
SUMMARY
The richness, abundance, diversity and functional composition of soil macrofauna were studied in two sites of the evergreen medium forest (Helechal and Vallecito), located at the Ecological Reserve El Salón from Sierra del Rosario; they were analyzed with respect to other land use systems evaluated in Cuba. The macrofauna was collected in situ from $25 \times 25 \times 20 \mathrm{~cm}$ monoliths extracted from the soil. In this type of ecosystem, 34 families of the edaphic macrofauna belonging to 18 orders were found. The Vallecito site showed higher families richness, density and diversity of the macrofauna (34 families, 1,298.5 individuals $\mathrm{m}^{-2}$ and $\left.\mathrm{H}^{\prime}=2.72\right)$ compared to Helechal (19 families, 749.2 individuals $\mathrm{m}^{-2}$ and $\mathrm{H}^{\prime}=2.57$ ). Although, only richness showed significant differences between the two locations. The seasonality in the sampling marked the variations between the sites, which was carried out during a rainy month in Helechal (October 2009) and during a dry month in Vallecito (March 2011). The functional composition of the edaphic community was similar in both sites, where the most outstanding guilds were litter detritivores, soil engineers and predators. However, the dominant families within each functional group changed between sites. The structure found of the macrofauna was very similar to those of other tropical forests, and hardly changed in relation to secondary forests studied in Cuba, although it did change in relation to more disturbed systems such as pastures and crops.
\end{abstract}

Key words: tropical evergreen forest, soil macroinvertebrates, functional diversity.

\section{RESUMEN}

Se estudiaron la riqueza, abundancia, diversidad y composición funcional de la macrofauna del suelo en dos sitios de bosque siempreverde medio (Helechal y Vallecito), ubicados en la Reserva Ecológica El Salón de la Sierra del Rosario; y se analizaron con respecto a otros sistemas de uso de la tierra evaluados en Cuba. La macrofauna fue recolectada in situ, de monolitos de $25 \times 25 \times 20 \mathrm{~cm}$ extraídos del suelo. Se encontraron en este tipo de ecosistema, 34 familias de la macrofauna edáfica pertenecientes a 18 órdenes. El sitio Vallecito mostró una mayor riqueza de familias, densidad y diversidad de la macrofauna (34 familias, $1.298,5$ individuos $\mathrm{m}^{-2}$ y $\left.H^{\prime}=2,72\right)$ en comparación con Helechal (19 familias, 749,2 individuos $\mathrm{m}^{-2} \mathrm{y} \mathrm{H}^{\prime}=2,57$ ), aunque solo la riqueza arrojó diferencias significativas entre las dos localidades. La estacionalidad en el muestreo marcó las variaciones entre los sitios, el cual se realizó en un mes de lluvia en Helechal (octubre de 2009) y en un mes de seca en Vallecito (marzo de 2011). La composición funcional de la comunidad edáfica fue similar en ambos sitios, donde los gremios más sobresalientes fueron los detritívoros de la hojarasca, los ingenieros del suelo y los depredadores. No obstante, las familias dominantes dentro de cada grupo funcional cambiaron entre sitios. La estructura encontrada de la macrofauna fue muy parecida a la de otros bosques tropicales, y apenas cambió con relación a bosques secundarios estudiados en Cuba, pero sí respecto a sistemas más perturbados como pastizales y cultivos.

Palabras clave: bosque siempreverde tropical, macroinvertebrados del suelo, diversidad funcional.

\section{INTRODUCCIÓN}

Los macroinvertebrados edáficos (mayores de $2 \mathrm{~mm}$ de diámetro) regulan los procesos físico-químicos del suelo, y son valorados como bioindicadores de calidad o altera- ción ambiental. Entre los grupos funcionales de la macrofauna que determinan el equilibrio y el funcionamiento del medio edáfico se pueden encontrar los ingenieros del suelo o del ecosistema, los detritívoros de la hojarasca, los herbívoros y los depredadores. Los ingenieros del ecosistema 
cambian la estructura física del terreno, los detritívoros ayudan en la fragmentación de la hojarasca y estimulan el proceso de descomposición, y los herbívoros y los depredadores controlan la disponibilidad de los recursos del hábitat (Ruiz et al. 2008).

La distribución de los invertebrados terrestres depende de varios factores, entre ellos, las precipitaciones o la estacionalidad del clima; que a la vez definen la temperatura y la humedad del suelo, de modo que son las variables edafo-climáticas que más influyen sobre estas comunidades en los suelos tropicales. Generalmente, es en la época lluviosa, y no en la de seca, donde se encuentra la mayor diversidad y abundancia de la fauna edáfica, pues el agua contribuye con procesos vitales de estos organismos como la respiración, reproducción y alimentación (Souza et al. 2016). No obstante, dicho patrón puede variar dentro del período lluvioso según las características del ecosistema y el tipo de suelo, que propician condiciones de exceso de agua; ante las cuales las comunidades edáficas disminuyen ya que salen a la superficie o migran a estratos profundos del suelo, en busca de micro-ambientes más apropiados para su establecimiento (Herrera et al. 1988). En una investigación realizada en bosques tropicales de diferentes edades en Brasil durante la época de seca, se encuentra una alta densidad de la macrofauna del suelo, mediada por el alto número de insectos sociales recolectados; resultados que se refieren coincidentes con otros obtenidos durante el período seco con respecto al período húmedo (Amazonas et al. 2018).

También la transformación de los ecosistemas naturales a agroecosistemas provoca cambios negativos en la composición y estructura de la macrofauna edáfica, producto del impacto directo sobre la cobertura vegetal y la estructura física del suelo. Las variaciones ocurridas en estas comunidades obedecen en primera instancia al cambio y a la intensidad del uso de la tierra, y además a variables edáficas condicionadas por la pérdida del componente vegetal y por la intensidad de manejo, tales como la temperatura, la humedad, la textura, el estatus nutricional y el contenido de materia orgánica (Ruiz et al. 2008). En comparación con otros ecosistemas, los bosques presentan una composición vegetal diversa y una amplia capa de hojarasca, la cual al depositarse en el suelo como mantillo crea un microclima favorable, y sirve de microhábitat y alimento para muchos organismos de la fauna edáfica (De la Rosa y Negrete-Yankelevich 2012). Diversos estudios en bosques del trópico húmedo exponen valores altos de riqueza taxonómica, diversidad y abundancia de la macrofauna con relación a otros usos de la tierra menos conservados, y también la dominancia de táxones como Hymenoptera, Isoptera, Oligochaeta, Diplopoda y Coleoptera (Rousseau et al. 2013, Marichal et al. 2014, Souza et al. 2016).

En los bosques maduros, los grupos funcionales de la macrofauna mayormente favorecidos son los detritívoros y los ingenieros del suelo. Principalmente los detritívoros, como organismos que se alimentan de residuos, son muy sensibles a la degradación (De la Rosa y NegreteYankelevich 2012). La abundancia y diversidad de estos grupos está asociada a la riqueza y abundancia de especies arbóreas, a una mayor cobertura vegetal, a una alta disponibilidad y calidad de la materia orgánica (Brévault et al. 2007). Durán y Suárez (2013) y Gómez et al. (2016) obtienen una estructura funcional definida en bosques y sistemas agroforestales del trópico sobre todo en la estación lluviosa, que responde a mayores valores de abundancia para detritívoros e ingenieros, y menores para depredadores y herbívoros; y lo justifican mediante la presencia de árboles que componen el dosel, la flora diversificada y la capa de hojarasca que mejora el hábitat y le da protección a esta fauna.

En Cuba, la mayoría de las investigaciones sobre la macrofauna edáfica se han desarrollado en ecosistemas seminaturales y altamente manejados, por ejemplo, en bosques secundarios, sistemas silvopastoriles, pastizales, fincas agropecuarias, cañaverales y cultivos varios, solo durante el período lluvioso (Cabrera Dávila et al. 2011ab, García et al. 2014). En estas investigaciones se hallan los mayores valores de riqueza taxonómica y abundancia en los sistemas con árboles y más estables en el manejo, y los menores en los sistemas de uso más perturbados. Por su parte, Cabrera Dávila et al. (2011b) analizan los grupos funcionales de ingenieros del ecosistema, detritívoros de la hojarasca, herbívoros y depredadores; y detectan una mayor abundancia de ingenieros y de detritívoros de la macrofauna en los ecosistemas boscosos, donde hay una mejor conservación del estrato de hojarasca y de la estructura del suelo.

A pesar de estos estudios en varios sistemas de uso de la tierra en Cuba, se cuenta con muy pocos resultados en bosques naturales conservados. Las publicaciones en este tipo de ambiente en el país, reflejan la composición y la estructura de la macrofauna, así como de los grupos claves de Oligochaeta, Diplopoda, Gastropoda y Coleoptera, en el macizo montañoso Sierra del Rosario, donde se realizó el presente estudio (González y Herrera 1983, González 1984, 1989, Martínez y Sánchez 2000). También Cabrera Dávila (2017) realiza la rectificación de la primera lista taxonómica de macroinvertebrados edáficos para el bosque siempreverde mesófilo de la región, la cual queda finalmente constituida por tres phyla, ocho clases y subclases, 21 órdenes, 33 familias identificadas, y a niveles inferiores se mantienen 13 géneros y ocho especies.

Teniendo en cuenta el escaso conocimiento de las comunidades de la macrofauna edáfica en bosques naturales y altamente conservados en Cuba, el presente trabajo persigue como objetivo fundamental determinar y comparar la riqueza de familias, la diversidad, abundancia y composición funcional de la macrofauna del suelo en dos sitios de bosque primario siempreverde dentro de la Reserva Ecológica El Salón en la Sierra del Rosario, que se estudian en diferentes períodos estacionales. Además, analizar estos resultados de la macrofauna con respecto a 
los obtenidos en otros ecosistemas con diferentes niveles de conservación/perturbación, evaluados anteriormente en el país. Se parte de las hipótesis que: a) las diferencias en cuanto a riqueza y abundancia de la macrofauna entre los dos sitios de bosque siempreverde en estudio responden a la estacionalidad y en específico a la humedad edáfica como factores que influyen sobre estas comunidades, y b) las comunidades de macroinvertebrados edáficos y en particular de organismos detritívoros son más abundantes y diversas en el ecosistema de bosque maduro siempreverde, con respecto a las que se encuentran en otros sistemas de uso de la tierra más alterados por el hombre.

\section{MÉTODOS}

Características del área de estudio. La Reserva de la Biosfera Sierra del Rosario pertenece a la Cordillera de Guaniguanico en el occidente de Cuba, y representa uno de los sistemas montañosos más relevantes en el país. La Sierra del Rosario se encuentra fuertemente diseccionada y entre sus formaciones montañosas está El Salón, declarada Reserva Ecológica y considerada área núcleo dentro de esta Reserva de la Biosfera. El Salón es, por ende, una de las zonas de mayor protección e interés conservacionista, donde se destaca el ecosistema de bosque siempreverde mesófilo tropical (Herrera et al. 1988).

Se seleccionaron para el estudio dos sitios en El Sa-

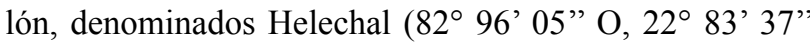
$\mathrm{N})$ y Vallecito $\left(82^{\circ} 58^{\prime} 17^{\prime \prime} \mathrm{O}, 22^{\circ} 49^{\prime} 54^{\prime \prime} \mathrm{N}\right)$ (coordenadas WGS84). La selección se basó en la amplia caracterización de la vegetación y los suelos en estos sitios y porque se cuenta con datos previos de la macrofauna para el bosque siempreverde en la zona (González y Herrera 1983, Herrera et al. 1988). Ambos sitios están ubicados a una altura aproximada de $450 \mathrm{~m}$ s.n.m., tienen exposición Norte y vegetación de bosque siempreverde medio, compuesta por árboles emergentes de hasta $25 \mathrm{~m}$ de altura, estrato arbóreo continuo con individuos de 6 hasta $20 \mathrm{~m}$ y sotobosque abierto con pocos arbustos de 1 a $3 \mathrm{~m}$ de altura (Herrera et al. 1988).

El sitio Helechal presentó buen estado de conservación y la vegetación estuvo constituida por un bosque siempreverde medio más húmedo que la de otros sitios de la Sierra del Rosario. Dominaron principalmente las especies de árboles: Calophyllum antillanum Britton, Dendropanax arboreus (L.) Decne. et Planch., Matayba oppositifolia (A. Rich.) Britton, Sideroxylon foetidissimum Jacq. subsp. foetidissimum, Prunus occidentalis Sw. y Prunus myrtifolia (L.) Urb. Además, producto de la alta humedad en el sitio, se encontró al helecho arborescente Cyathea arborea (L.) Sm., cuyos individuos alcanzaron hasta $10 \mathrm{~m}$ de altura (Herrera et al. 1988).

El sitio Vallecito manifestó una etapa madura y poco alterada del bosque siempreverde medio, y entre las especies arbóreas más notables coincidieron algunas mencionadas para el sitio anterior: Bursera simaruba (L.) Sarg., Calo- phyllum antillanum Britton, Dendropanax arboreus (L.) Decne. et Planch., Talipariti elatum (Sw.) Fryxell, Matayba oppositifolia (A. Rich.) Britton, Oxandra lanceolata (Sw.) Baill., Prunus occidentalis Sw., Pseudolmedia spuria (Sw.) Griseb., Trophis racemosa (L.) Urb. y Zanthoxylum martinicense (Lam.) DC. (Herrera et al. 1988). Solo en este sitio se detallaron algunas características de la vegetación, especialmente de las especies mejor representadas, y se tomaron como referencia para describir la estructura del ecosistema de bosque siempreverde medio en la Sierra del Rosario; la cual fue similar a la de otros bosques tropicales de Guyana, Costa de Marfil, Panamá, y sobre todo respondió a la del bosque tipo insular (Herrera et al. 1988). Las especies más abundantes de este sitio como $P$. spuria (1.175 individuos ha $\left.{ }^{-1}\right)$, T. racemosa $\left(692\right.$ individuos ha ${ }^{-1}$ ) y $O$. lanceolata (625 individuos ha $\left.{ }^{-1}\right)$ presentaron diámetros menores de $20 \mathrm{~cm}$, que las especies menos dominantes de M. oppositifolia (333 individuos ha$\left.{ }^{1}\right)$, D. arboreus (133 individuos ha ${ }^{-1}$ ) y T. elatum (108 individuos ha ${ }^{-1}$ ), que tuvieron entre 25 y $40 \mathrm{~cm}$ de diámetro. Asimismo, P. spuria, M. oppositifolia y T. elatum fueron los árboles con mayor altura, entre 20 y $25 \mathrm{~m}$, y en particular las dos primeras tuvieron aportes superiores al área basal del bosque siempreverde medio con $9,06 \mathrm{~m}^{2} \mathrm{ha}^{-1} \mathrm{y}$ $6,08 \mathrm{~m}^{2} \mathrm{ha}^{-1}$ respectivamente; mientras que $T$. racemosa, $O$. lanceolata y $D$. arboreus no sobrepasaron los $15 \mathrm{~m}$ de altura, y estas especies junto a T. elatum contribuyeron con valores inferiores al área basal, entre 1,26 y $3,19 \mathrm{~m}^{2} \mathrm{ha}^{-1}$.

Durante la investigación en los años 2009 y 2011, hubo poca variación en la región de la Sierra del Rosario con respecto a los estimados históricos (medidos de 1970 a 1987) de temperatura media del aire y de precipitación total anuales que fueron de $24,4{ }^{\circ} \mathrm{C}$ y $2.014 \mathrm{~mm}$, respectivamente (Herrera et al. 1988). Asimismo, se mantuvieron valores mínimos de temperatura y precipitación durante los meses de noviembre a abril y máximos de mayo a octubre, con una pronunciada estación de seca desde diciembre hasta marzo (figura 1).

El suelo más representativo de toda la localidad, El Salón, fue definido como Fersialítico Pardo Amarillento, con características de menor acidez y mayor humificación y fertilidad, en comparación con otros de la Reserva de la Biosfera Sierra del Rosario (Herrera et al. 1988). Este suelo se correspondió con un Cambisol Mólico de acuerdo a la clasificación de la World Reference Base (Driessen et al. 2001), y con un Fersialítico Amarillento (FrsA) según la última clasificación de los suelos de Cuba (Hernández et al. 2015). En los sitios de estudio en El Salón, se analizaron algunas características edáficas de los primeros $20 \mathrm{~cm}$ de profundidad, las cuales manifestaron menores tenores de materia orgánica, arena y arcilla y mayores de limo y capacidad de retención de agua en Helechal (materia orgánica: 5,1 \%, arena: $33,5 \%$, limo: $38,0 \%$, arcilla: $26,5 \%$, capacidad de retención de agua [CRA]: $36,7 \%$ ), con respecto a Vallecito (materia orgánica: 10,6 \%, arena: 41,3\%, limo: 13,0\%, arcilla: 45,7 \%, CRA: 31,1 \%) (Sánchez et al. 2012). 


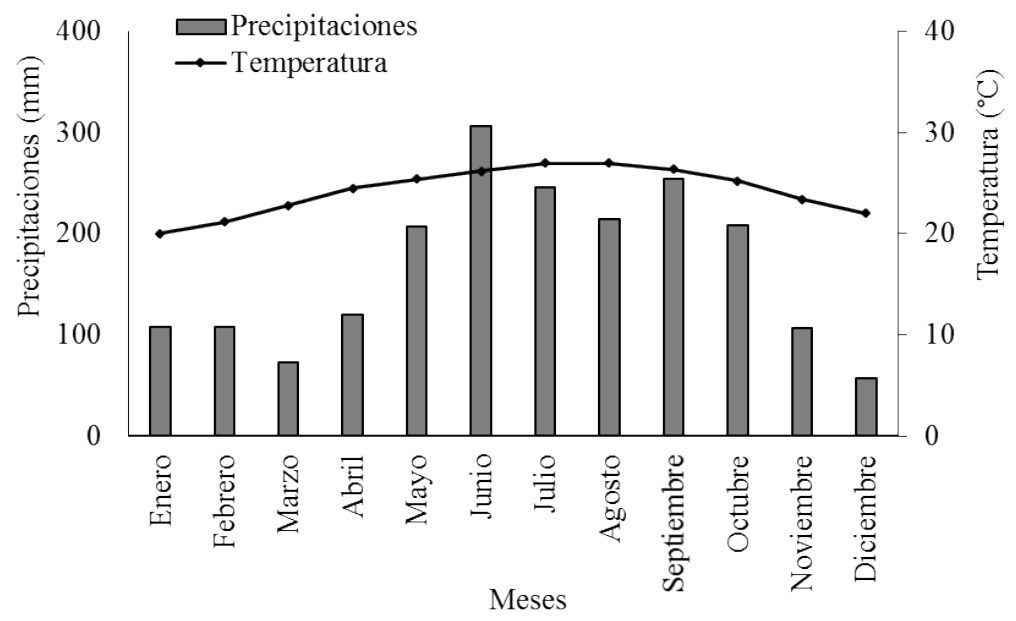

Figura 1. Comportamiento histórico de las precipitaciones y las temperaturas (de 1970 a 1987, Herrera et al. 1988) durante los meses del año en El Salón, Sierra del Rosario, Cuba.

Historical behavior of rainfall and temperatures (from 1970 to 1987, Herrera et al. 1988) during the months of the year at El Salón, Sierra del Rosario, Cuba.

Muestreo y procesamiento de la macrofauna. El muestreo de la macrofauna edáfica se efectuó en octubre de 2009 en el sitio Helechal (mes de lluvia) y en marzo de 2011 en Vallecito (mes de seca). Las fechas de muestreo se corresponden con los períodos en que se pudo tener acceso a las áreas. La macrofauna se recolectó de monolitos de suelo de $25 \times 25 \times 20 \mathrm{~cm}$, según la Metodología del Programa de Investigación Internacional "Biología y Fertilidad del Suelo Tropical" o TSBF (Anderson e Ingram 1993). En el centro de cada sitio, se trazaron tres transectos paralelos de $50 \mathrm{~m}$ de longitud, separados entre sí también en $50 \mathrm{~m}$. Se procesaron dos monolitos extraídos de los extremos de cada transecto, para un total de seis monolitos estudiados por sitio. De estos monolitos, los invertebrados fueron recolectados manualmente in situ y preservados en alcohol $75 \%$, excepto las lombrices que se conservaron en formol $4 \%$.

La macrofauna se identificó y caracterizó hasta el nivel taxonómico de familia. En este estudio no se tomaron en cuenta algunos grupos incluidos en la lista taxonómica recién publicada para El Salón en la Sierra del Rosario (Cabrera Dávila 2017), como por ejemplo: Diplura, Pseudoscorpionida y Schizomida, ya que fueron muy raros y, por lo general, no son capturados mediante la metodología descrita.

Para cada monolito se estimó la riqueza taxonómica (número de familias) y la densidad (abundancia de individuos expresada como individuos $\mathrm{m}^{-2}$ ), para toda la macrofauna y para los distintos gremios funcionales. La clasificación funcional se realizó de acuerdo con Cabrera Dávila et al. (2011b), que incluyó: a) ingenieros del suelo, integrados por las lombrices de tierra, las termitas y las hormigas, los dos primeros organismos descomponedores y habitantes del interior del suelo y los últimos, fundamentalmente de hábito trófico omnívoro; b) detritívoros de la hojarasca, que agrupó a los táxones consumidores de materia orgánica semidescompuesta de origen vegetal y animal (hojarasca), y presentes en la superficie y primeros cinco centímetros del suelo; c) herbívoros, los cuales comprendieron los grupos que se alimentan de las partes vivas de las plantas; y d) depredadores, que se alimentan de pequeños animales vivos.

Análisis estadísticos. Para las variables de riqueza y abundancia se calcularon la media y la desviación estándar (DE). Se construyeron las curvas de rango/abundancia a partir del $\log _{10}$ de la abundancia relativa (AR) de las familias encontradas de la macrofauna, para analizar la dominancia y diversidad. Además, se calculó el índice de diversidad de Shannon-Wiener (H'). Debido a que los datos no cumplieron las premisas paramétricas, se realizaron análisis de varianza no paramétricos de una vía (PERMANOVA) con base a 9.999 permutaciones y con el empleo de una matriz de distancia euclidiana, para comprobar las posibles diferencias de la riqueza de familias y la abundancia por monolito, entre los dos sitios estudiados en distintas épocas de muestreo. Esta misma prueba se utilizó, con la prueba a posteriori Corrección de Bonferroni, para comparar la abundancia y la riqueza entre gremios funcionales, y la abundancia entre las familias de cada gremio en los diferentes sitios. El índice de diversidad y los análisis estadísticos se obtuvieron a través del software PAST versión 3.15, y el diagrama de cajas mediante el programa InfoStat versión 2012.

\section{RESULTADOS}

En total se registraron 34 familias de la macrofauna edáfica (19 en Helechal y 34 en Vallecito) pertenecientes a 18 órdenes. La comparación del número medio de familias 
por monolito arrojó diferencias significativas $(P=0,011)$ entre los sitios estudiados de Helechal $(12,7 \pm 2,5)$ y Vallecito $(18,2 \pm 3,7)$. Sin embargo, las diferencias en la densidad promedio entre Helechal $(749,2 \pm 217,3$ individuos $\mathrm{m}^{-2}$ ) y Vallecito $\left(1.298,5 \pm 811,9\right.$ individuos $\left.\mathrm{m}^{-2}\right)$ no fueron significativas $(P=0,092)$.
En Vallecito hubo un predominio de las familias de insectos sociales de termitas (Termitidae) y hormigas (Formicidae) con relación a las demás familias de la macrofauna, según se apreció en las curvas de rango/abundancia (figura 2). Asimismo, se observaron en esta área como grupos menos abundantes, principalmente a hemípteros (Cyd-

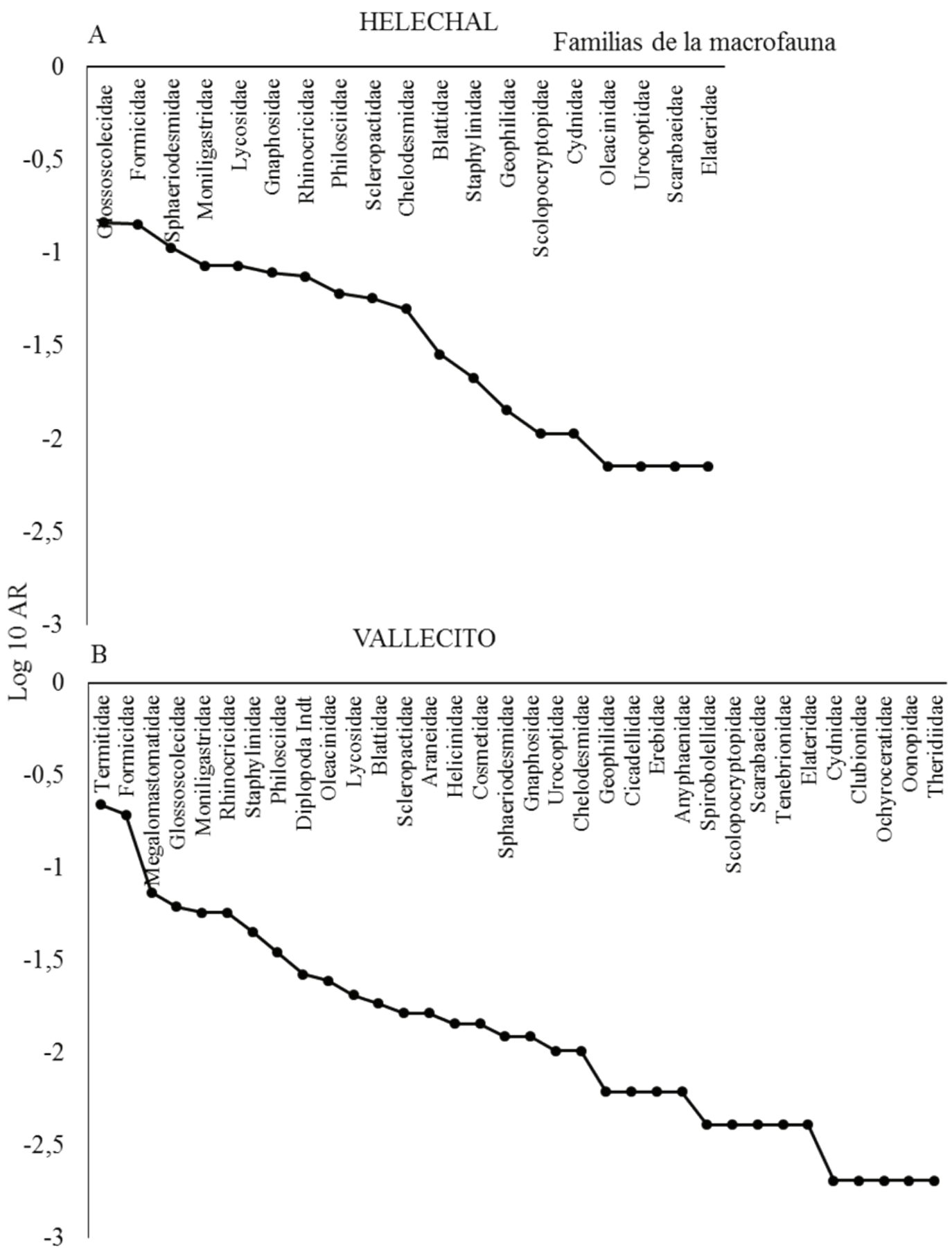

Figura 2. Curvas de rango/abundancia de la macrofauna edáfica en los sitios Helechal (A) y Vallecito (B) en El Salón, Sierra del Rosario, Cuba. Diplopoda Indt: Familia de milpié indeterminada.

Range/Abundance curves of the soil macrofauna in Helechal (A) and Vallecito (B) sites at El Salón, Sierra del Rosario, Cuba. Diplopoda Indt: Undetermined millipede family. 
nidae) y arañas (Clubionidae, Ochyroceratidae, Oonopidae, Theridiidae). En Helechal, prevaleció la familia de la lombriz de tierra Glossoscolecidae, seguida de formícidos, pero no se detectaron termitas. En este mismo sitio, los táxones de más baja abundancia estuvieron representados por caracoles (Oleacinidae y Urocoptidae) y escarabajos (Scarabaeidae y Elateridae). Las curvas de rango/abundancia reflejaron, además, una mayor equitatividad de las comunidades de la macrofauna en Helechal y un menor número de familias; mientras que en Vallecito hubo mayor riqueza, pero la abundancia se concentró en dos grupos, que acumularon $41 \%$ de los individuos frente a $29 \%$ en Helechal (figura 2A y 2B). Como consecuencia, el índice de diversidad de Shannon fue similar en ambos sitios de estudio, de 2,57 en Helechal y 2, 72 en Vallecito.

En cada sitio, las diferencias entre grupos funcionales respecto a la abundancia media por monolito, fueron significativas (Helechal: $P<0,001$ y Vallecito: $P=0,002$ ). En Helechal dominaron con una abundancia muy parecida los detritívoros de la hojarasca (288 individuos $\mathrm{m}^{-2}$ ) y los ingenieros del suelo (280 individuos $\mathrm{m}^{-2}$ ), una densidad intermedia tuvieron los depredadores $\left(168\right.$ individuos $\left.\mathrm{m}^{-2}\right) \mathrm{y}$ una muy baja los herbívoros (13,3 individuos $\mathrm{m}^{-2}$ ) (figura 3A). En Vallecito el patrón fue similar, de manera que la abundancia fue muy baja en herbívoros (ingenieros del suelo: 690,6 individuos $\mathrm{m}^{-2}$; detritívoros de la hojarasca: 368 individuos $\mathrm{m}^{-2}$; depredadores: 210,6 individuos $\mathrm{m}^{-2}$; herbívoros: 29,3 individuos $\mathrm{m}^{-2}$ ) (figura 3B). Igualmente, en cada sitio la riqueza media fue diferente entre gremios (Helechal y Vallecito: $P<0,001)$. En Helechal sobresalió la riqueza de detritívoros de la hojarasca (media 5, total 7 familias), seguido por los depredadores (media 4,16, total 7 familias) y con menor riqueza los ingenieros del suelo (media 2,83, total 3 familias) y los herbívoros (media 0,66 , total 2 familias). En Vallecito, se repitió el mismo patrón de diferencias en riqueza entre los distintos gremios (detritívoros de la hojarasca: media 7, total 12 familias; depredadores: media 6,5, total 14 familias; ingenieros del suelo: media 3,16, total 4 familias; herbívoros: media 1,5, total 4 familias) (figura 3).

Para dos de los cuatro grupos funcionales determinados, la abundancia varió significativamente entre familias en los diferentes sitios (Helechal: $P=0,044$ en detritívoros de la hojarasca y $P=0,006$ en depredadores; Vallecito: $P=0,023$ en detritívoros de la hojarasca y $P=0,002$ en depredadores). La excepción para ambas áreas boscosas, fueron los ingenieros del suelo (Helechal: $P=0,604$ y Vallecito: $P=0,841)$ y los herbívoros (Helechal: $P=0,960$ y Vallecito: $P=0,561)$. Las familias dominantes en cada gremio variaron entre los sitios estudiados (cuadro 1). En Helechal las familias más abundantes de ingenieros del suelo fueron Glossoscolecidae y Formicidae, para los detritívoros de la hojarasca sobresalieron Rhinocricidae y Sphaeriodesmidae, para los herbívoros Cydnidae y en cuanto a los depredadores se distinguieron Gnaphosidae y Lycosidae. En Vallecito las familias más dominantes estuvieron inte- gradas por Termitidae y Formicidae entre los ingenieros del suelo, Megalomastomatidae y Rhinocricidae entre los detritívoros de la hojarasca, Scarabaeidae entre los herbívoros y Staphylinidae entre los depredadores (cuadro 1).

\section{DISCUSIÓN}

El estudio en los dos sitios de bosque siempreverde medio en El Salón, Sierra del Rosario, revela la misma estructura funcional, pero una mayor riqueza y abundancia en Vallecito con respecto a Helechal, aunque solo la riqueza mostró diferencias significativas entre ellos. Los resultados sobre riqueza y abundancia pueden estar asociados al diferente período de muestreo, pues en Helechal se realiza al final de la época de mayor pluviosidad (octubre), con un gran acumulado de lluvias (figura 1), mientras que en Vallecito se efectúa al final de la época de seca (marzo). Es posible que una excesiva humedad en el suelo, establecida durante el período de máximas precipitaciones y condicionada por las características edáficas de alta capacidad de retención de agua y alto contenido de limo en el sitio Helechal (CRA: 36,7\%, limo: $38 \%$ ) (Sánchez et al. 2012), no sea beneficiosa para el desarrollo de las comunidades de invertebrados edáficos, influyendo en un resultado más bajo de riqueza y abundancia en esta localidad. También Hernández y Sánchez (2012) en un estudio realizado sobre la dinámica de la humedad del suelo principalmente en ecosistemas boscosos de la Sierra del Rosario, reportan valores altos de humedad a la profundidad de $0-5 \mathrm{~cm}$ del suelo para el sitio Helechal, durante los respectivos meses lluviosos (24-37 \%). Además, la presencia de helechos en este sitio, propios de lugares muy húmedos, es otra evidencia para tal argumentación (Herrera et al. 1988). Por otra parte, esta disminución de la macrofauna en Helechal, sobre todo en riqueza, puede responder adicionalmente al contenido de materia orgánica del suelo en este sitio $(5,1 \%)$, que aunque alto es menor que en Vallecito (10,6 \%) (Sánchez et al. 2012). La materia orgánica es importante para mantener la actividad de la biota en el suelo, y actúa como la principal fuente energética para diversificar las comunidades edáficas (De la Rosa y Negrete-Yankelevich 2012).

Estos resultados alcanzados en Helechal se corresponden con los enunciados anteriormente por González y Herrera (1983) y Herrera et al. (1988), quienes han descrito una caída brusca de la riqueza y abundancia de las comunidades del suelo en la época lluviosa para el mismo sitio de estudio en la Sierra del Rosario. Dichos autores discuten que las intensas lluvias determinan la migración de los individuos en busca de mejores condiciones de humedad, y comentan el efecto negativo que tiene sobre la edafofauna el lavado producido por el escurrimiento superficial durante este período en las montañas.

Otra diferencia entre los sitios de estudio es el mayor predominio de los insectos sociales en el caso de Vallecito, donde las hormigas y termitas representaron aproximadamente la mitad de la densidad de la macrofauna 
total (535,9 de 1.298,5 individuos $\mathrm{m}^{-2}$ ), mientras que en Helechal las termitas no se encontraron. Esta diferencia puede deberse a las condiciones de sequía en el muestreo de Vallecito en marzo, ya que existen reportes sobre el aumento de la riqueza y abundancia, principalmente de las termitas, en bosques tropicales húmedos durante el período seco (Teixeira 2007). Su decrecimiento rápido durante las lluvias y su permanencia y proliferación en la estación de seca se debería a la mudanza vertical de estos insectos en el perfil del suelo, huyendo de las inundaciones provocadas por las lluvias. Tanto para las termitas como para las hormigas, se señalan varios factores que influyen en la variación estacional de sus abundancias, dando gran significación a la cantidad de luz solar y al aumento de

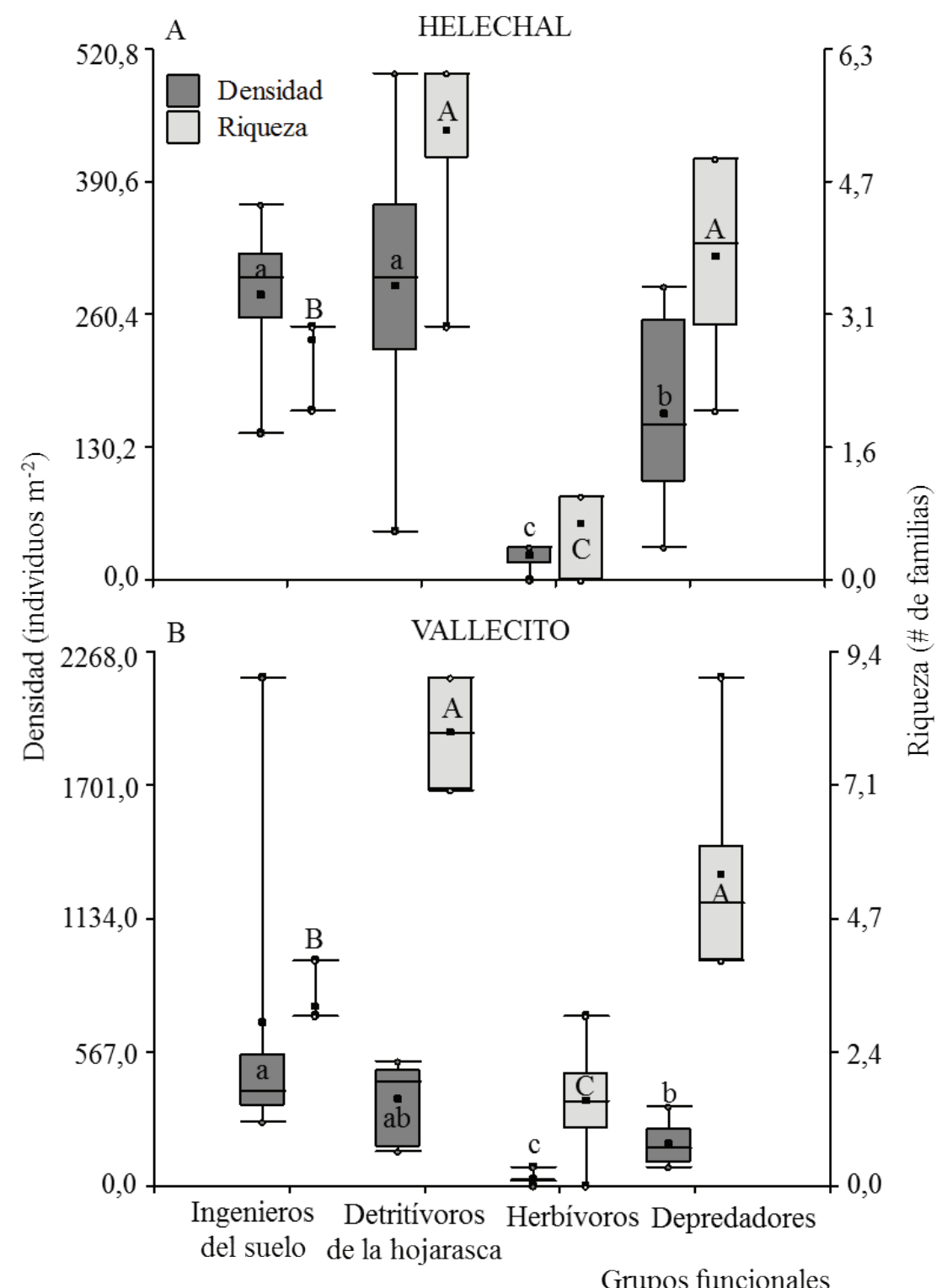

Figura 3. Diagrama de cajas de la Densidad y la Riqueza de los grupos funcionales de la macrofauna edáfica en los sitios Helechal (A) y Vallecito (B) en El Salón, Sierra del Rosario, Cuba. Letras distintas, minúsculas para la Densidad y mayúsculas para la Riqueza, indican diferencias significativas entre grupos funcionales dentro de cada sitio, por la prueba de Corrección de Bonferroni $(P<0,05)$. El diagrama muestra medianas (líneas en el interior de las cajas), medias (puntos negros), percentiles 25 y 75 (bordes inferior y superior de las cajas) y valores extremos (límites de líneas verticales) de ambas variables.

Boxplots of Density and Richness of soil macrofauna functional groups in Helechal (A) and Vallecito (B) sites at El Salón, Sierra del Rosario, Cuba. Different letters, lowercase letters for Density and uppercase letters for Richness, indicate significant differences among functional groups within each site, by the Bonferroni Correction test $(P<0.05)$. The boxplots show medians (lines inside the boxes), means (black dots), percentiles 25 and 75 (lower and upper edges of the boxes) and extreme values (vertical lines limits) of both variables. 
Cuadro 1. Densidad (media \pm DE) de las familias de la macrofauna pertenecientes a cada grupo funcional, en los sitios Helechal y Vallecito en El Salón, Sierra del Rosario, Cuba.

Rosario, Cuba.

Density (mean \pm SD) of the macrofauna families belonging to each functional group, in Helechal and Vallecito sites at El Salón, Sierra del

\begin{tabular}{|c|c|c|c|c|}
\hline \multirow{2}{*}{ Grupo funcional } & \multirow{2}{*}{ Nombre común } & \multirow{2}{*}{ Familia } & \multicolumn{2}{|c|}{ Densidad (individuos $\mathrm{m}^{-2}$ ) } \\
\hline & & & Helechal & Vallecito \\
\hline \multirow{4}{*}{ Ingenieros del suelo } & \multirow{2}{*}{ Lombrices de tierra } & Glossoscolecidae & $109,3 \pm 73,9$ ns & $80,0 \pm 83,4^{\mathrm{ns}}$ \\
\hline & & Moniligastridae & $64,0 \pm 36,5$ & $74,6 \pm 55,1$ \\
\hline & Hormigas & Formicidae & $106,6 \pm 118,7$ & $250,6 \pm 114,8$ \\
\hline & Termitas & Termitidae & $0,0 \pm 0,0$ & $285,3 \pm 683,4$ \\
\hline \multirow{12}{*}{ Detritívoros de la hojarasca } & \multirow{3}{*}{ Caracoles } & Helicinidae & $0,0 \pm 0,0 \mathrm{c}$ & $18,6 \pm 15,8 \mathrm{c}$ \\
\hline & & Megalomastomatidae & $0,0 \pm 0,0 \mathrm{c}$ & $96,0 \pm 108,1 \mathrm{a}$ \\
\hline & & Urocoptidae & $5,3 \pm 13,1 \mathrm{c}$ & $13,3 \pm 15,8 \mathrm{~d}$ \\
\hline & \multirow{2}{*}{ Cochinillas } & Philosciidae & $45,3 \pm 58,5 \mathrm{~b}$ & $45,3 \pm 54,9 \mathrm{~b}$ \\
\hline & & Scleropactidae & $42,6 \pm 74,1 \mathrm{~b}$ & $21,3 \pm 24,1 \mathrm{c}$ \\
\hline & \multirow{5}{*}{ Milpiés } & Chelodesmidae & $37,3 \pm 26,1 \mathrm{~b}$ & $13,3 \pm 15,8 \mathrm{~d}$ \\
\hline & & Rhinocricidae & $56,0 \pm 33,2 \mathrm{a}$ & $74,6 \pm 77,5 \mathrm{a}$ \\
\hline & & Sphaeriodesmidae & $80,0 \pm 50,6 \mathrm{a}$ & $16,1 \pm 39,2 \mathrm{c}$ \\
\hline & & Spirobolellidae & $0,0 \pm 0,0 \mathrm{c}$ & $5,3 \pm 8,3 \mathrm{e}$ \\
\hline & & Diplopoda Indt & $0,0 \pm 0,0 \mathrm{c}$ & $34,6 \pm 38,4 b$ \\
\hline & Cucarachas & Blattidae & $21,3 \pm 13,1 \mathrm{~b}$ & $24,0 \pm 22,1 \mathrm{c}$ \\
\hline & Escarabajos & Tenebrionidae & $0,0 \pm 0,0 \mathrm{c}$ & $5,3 \pm 8,3 \mathrm{e}$ \\
\hline \multirow{4}{*}{ Herbívoros } & Escarabajos & Scarabaeidae & $5,3 \pm 8,3$ ns & $10,6 \pm 19,4 \mathrm{~ns}^{\mathrm{ns}}$ \\
\hline & \multirow{2}{*}{ Chinches y salta hojas } & Cicadellidae & $0,0 \pm 0,0$ & $8,0 \pm 8,7$ \\
\hline & & Cydnidae & $8,0 \pm 13,4$ & $2,6 \pm 6,6$ \\
\hline & Orugas & Erebidae & $0,0 \pm 0,0$ & $8,0 \pm 8,7$ \\
\hline \multirow{14}{*}{ Depredadores } & Caracoles & Oleacinidae & $5,3 \pm 8,3 \mathrm{c}$ & $32,0 \pm 20,2 \mathrm{~b}$ \\
\hline & \multirow{2}{*}{ Ciempiés } & Geophilidae & $10,6 \pm 19,4 \mathrm{~b}$ & $8,0 \pm 8,7 \mathrm{c}$ \\
\hline & & Scolopocryptopidae & $8,0 \pm 13,4 \mathrm{c}$ & $5,3 \pm 13,1 \mathrm{c}$ \\
\hline & \multirow{2}{*}{ Escarabajos } & Elateridae & $5,3 \pm 8,3 \mathrm{c}$ & $5,3 \pm 8,3 \mathrm{c}$ \\
\hline & & Staphylinidae & $16,0 \pm 10,1 \mathrm{~b}$ & $58,6 \pm 62,9 \mathrm{a}$ \\
\hline & \multirow{8}{*}{ Arañas } & Anyphaenidae & $0,0 \pm 0,0 \mathrm{c}$ & $8,0 \pm 13,4 \mathrm{c}$ \\
\hline & & Araneidae & $0,0 \pm 0,0 \mathrm{c}$ & $21,3 \pm 19,4 b$ \\
\hline & & Clubionidae & $0,0 \pm 0,0 \mathrm{c}$ & $2,6 \pm 6,5 \mathrm{c}$ \\
\hline & & Gnaphosidae & $58,6 \pm 53,2 \mathrm{a}$ & $16,0 \pm 20,2 \mathrm{~b}$ \\
\hline & & Lycosidae & $64,0 \pm 55,4 \mathrm{a}$ & $26,6 \pm 31,5 b$ \\
\hline & & Ochyroceratidae & $0,0 \pm 0,0 \mathrm{c}$ & $2,6 \pm 6,5 \mathrm{c}$ \\
\hline & & Oonopidae & $0,0 \pm 0,0 \mathrm{c}$ & $2,6 \pm 6,5 \mathrm{c}$ \\
\hline & & Theridiidae & $0,0 \pm 0,0 \mathrm{c}$ & $2,6 \pm 6,5 \mathrm{c}$ \\
\hline & Opiliones & Cosmetidae & $0,0 \pm 0,0 \mathrm{c}$ & $18,6 \pm 6,5 \mathrm{~b}$ \\
\hline
\end{tabular}

Diplopoda Indt: Familia de milpié indeterminada.

Valores promedio con letras distintas indican diferencias significativas entre familias de cada grupo funcional dentro de cada sitio, por la prueba de Corrección de Bonferroni $(P<0,05)$. ns: no significativo $(P>0,05)$. 
la temperatura del suelo para un óptimo desenvolvimiento larval y actividad de forrajeo de las obreras y también a la disponibilidad del recurso alimentario (Teixeira 2007). Igualmente, Amazonas et al. (2018) revelan la superioridad de los insectos sociales de termitas y hormigas durante el período seco con respecto al período húmedo, en los ecosistemas de bosques.

La alta diversidad apreciada en Helechal $\left(H^{\prime}=2,57\right)$ y Vallecito $\left(H^{\prime}=2,72\right)$ (figura 2), es comúnmente alcanzada en escenarios con características semejantes, debido a la elevada cantidad de hojarasca y la materia orgánica almacenadas en ellos. Para el área estudiada, se muestran valores altos de materia orgánica en el suelo (Sánchez et al. 2012), así como un considerable aporte de hojarasca en el bosque, que es de $10 \mathrm{Mg} \mathrm{ha}^{-1}$ año $^{-1}$, lo que garantiza una constante fuente de materia orgánica que tapiza el suelo del ecosistema (Herrera et al. 1988). De la Rosa y Negrete-Yankelevich (2012) plantean que los bosques mesófilos maduros sostienen una gran complejidad vegetal y un elevado grado de endemismo que genera mosaicos de hojarascas y un mejor reparto de recursos, lo cual origina la coexistencia de una comunidad edáfica muy heterogénea y numerosa, a la vez que reduce la presión competitiva entre los macroinvertebrados del suelo.

Comparando con observaciones desarrolladas en hábitats conservados en Cuba, la dominancia de insectos sociales y de lombrices en los sitios estudiados coincide con los grupos dominantes detectados para los bosques siempreverdes investigados anteriormente en la Sierra del Rosario. González y Herrera (1983) y Herrera et al. (1988) para esta región determinan como grupos de la macrofauna más abundantes a las hormigas (Hymenoptera, Formicidae), las lombrices de tierra (Oligochaeta), los escarabajos (Coleoptera) y los milpiés (Diplopoda). Por otra parte, González (1984, 1989) y Martínez y Sánchez (2000) registran en la zona, específicamente para Diplopoda, Coleoptera y Oligochaeta a las familias dominantes de Sphaeriodesmidae, Staphylinidae y Glossoscolecidae, respectivamente, como se encontró también en esta investigación. No obstante, la abundancia total de la macrofauna del suelo de este trabajo es mucho menor que la expuesta por algunos de los autores ya mencionados. En el mismo bosque siempreverde estacional de la Sierra del Rosario, González y Herrera (1983) informan una densidad promedio mensual muy elevada, de 9.865 individuos $\mathrm{m}^{-2}$, que está determinada por la alta abundancia de formícidos que representan el $89 \%$ de la macrofauna total. La desigualdad entre estos resultados, podría deberse a la estabilidad en el muestreo desarrollado durante varios meses por estos autores, frente a una menor intensidad de muestreo aplicada en el presente estudio. También es probable, que los muestreos efectuados por González y Herrera (1983) hayan estado afectados por la distribución altamente agregada de las hormigas, tomando las muestras cercanas a hormigueros y derivando en una alta densidad de estos insectos y de la macrofauna total (Amazonas et al. 2018).
Investigaciones en Cuba para otros sistemas de uso de la tierra con diferentes niveles de perturbación, revelan valores menores de riqueza de familias y de densidad promedio de la macrofauna en la mayoría de los sistemas, con relación a los bosques conservados de este estudio. Cabrera Dávila et al. (2011a) examinan bosques secundarios, pastizales, cultivos varios y cañaverales ubicados en las provincias Artemisa y Mayabeque de la región occidental del país, y encuentran en los bosques secundarios igual número de familias (33) que en los bosques primarios siempreverdes del presente estudio, que es claramente superior al de los pastizales (18) los cultivos varios (16) y los cañaverales (15). Igualmente, la densidad promedio de la macrofauna total para los bosques secundarios es similar a la hallada en los bosques de El Salón en la Sierra del Rosario $\left(1.166,60\right.$ individuos $\left.\mathrm{m}^{-2}\right)$, pero los restantes sistemas de uso muestran resultados considerablemente menores (pastizales: 581 individuos $\mathrm{m}^{-2}$, cultivos varios: 294 individuos $\mathrm{m}^{-2}$, cañaverales: 280 individuos $\mathrm{m}^{-2}$ ). Por su parte, García et al. (2014) valoran los sistemas de cultivos varios, fincas agropecuarias, sistemas silvopastoriles y pastizales en la provincia Matanzas (del occidente de Cuba), y hallan la misma tendencia de resultados, con mayores valores de riqueza taxonómica y abundancia en los sistemas menos perturbados de silvopastoril y pastizal. Ambos trabajos explican que la riqueza taxonómica y la densidad de la macrofauna se afectan en función de la intensidad del uso de la tierra y del nivel de degradación del medio edáfico (Cabrera Dávila et al. 2011a, García et al. 2014).

En otros ambientes tropicales se han encontrado patrones similares de reducción de la diversidad entre bosques y sistemas agroforestales, pastizales y cultivos (Rousseau et al. 2013 en Nicaragua, Marichal et al. 2014 en la Amazonia brasileña, Souza et al. 2016 en la Mata Atlántica de Brasil). El mismo esquema se repite para la abundancia, que en medios forestales y agroforestales oscila en los trabajos ya citados entre 293,3 y 1.400 individuos $\mathrm{m}^{-2}$, pero baja en pastizales a valores entre 279,4 y 800 individuos $\mathrm{m}^{-2}$, y en medios agrícolas a densidades entre 176,9 y 400 individuos $\mathrm{m}^{-2}$. También en coincidencia con la investigación en curso, dichos autores señalan como grupos más abundantes, sobre todo en los bosques, a las hormigas $\left(254,2\right.$ - 400,3 individuos $\left.\mathrm{m}^{-2}\right)$ y las termitas $(133,3-309,3$ individuos $\mathrm{m}^{-2}$ ).

La estructura funcional de la macrofauna, resultó casi la misma en los dos sitios estudiados, pues en ambos hubo un mayor número de individuos y de familias correspondientes a los gremios de detritívoros de la hojarasca, ingenieros del suelo y depredadores, y menores valores en el caso de los herbívoros (figura 3). Tales resultados son bastante congruentes con los alcanzados en otros estudios cubanos. Cabrera Dávila et al. (2011b) encuentran en el país en las áreas más conservadas de bosques secundarios, como en los bosques siempreverdes en estudio, una dominancia de detritívoros (22 familias y 701 individuos $\mathrm{m}^{-2}$ ) y de ingenieros del suelo (4 familias y 320 individuos $\mathrm{m}^{-2}$ ) 
con respecto a depredadores ( 9 familias y 96 individuos $\mathrm{m}^{-2}$ ) y herbívoros (5 familias y 38 individuos $\mathrm{m}^{-2}$ ); pero para otros sistemas de uso más perturbados como los pastizales y los cultivos varios esta estructura cambia, pues por lo general hay mayores cantidades de ingenieros (pastizales: 4 familias y 386 individuos $\mathrm{m}^{-2}$, cultivos varios: 2 familias y 179 individuos $\mathrm{m}^{-2}$ ) y de herbívoros (pastizales: 3 familias y 116 individuos $\mathrm{m}^{-2}$, cultivos varios: 9 familias y 36 individuos $\mathrm{m}^{-2}$ ).

Para diferentes ecosistemas del trópico húmedo, también hay registrados resultados semejantes de composición funcional. Durán y Suárez (2013) totalizan en orden decreciente entre los sistemas agroforestales de la Amazonia colombiana: de 210 a 427 individuos $\mathrm{m}^{-2}$ de ingenieros, de 39 a 97 individuos $\mathrm{m}^{-2}$ de detritívoros, de 6 a 65 individuos $\mathrm{m}^{-2}$ de herbívoros y de 8 a 65 individuos $\mathrm{m}^{-2}$ de depredadores; y de igual manera Gómez et al. (2016) en ecosistemas naturales de Argentina confirman una mayor afectación en la abundancia de depredadores ( 7 individuos $\mathrm{m}^{-2}$ ) y de herbívoros (19 individuos $\mathrm{m}^{-2}$ ).

Diversos autores exponen que, en ecosistemas estables con una menor perturbación sobre el suelo y una continua cubierta de hojarasca, como ocurre en los ecosistemas en estudio (los bosques), existen mayores abundancias de detritívoros y depredadores y menores poblaciones de herbívoros; mientras que los herbívoros y algunos ingenieros del ecosistema (lombrices y termitas) se asocian más a los pastizales con altas densidades de raíces (Brown et al. 2001). Principalmente, las comunidades de detritívoros e ingenieros del suelo se han relacionado con el mayor aporte, calidad (baja relación Carbono/Nitrógeno) y grado de humedad de la hojarasca, y con el nivel de macronutrientes y fertilidad del suelo (Brévault et al. 2007, Zerbino et al. 2008).

Las familias más abundantes dentro de cada grupo funcional (cuadro 1), concuerdan en la mayoría de casos con las que se mencionan en la literatura internacional. Brévault et al. (2007) refieren para sistemas subtropicales a Formicidae $(53,6 \%)$ y Termitidae $(24,7 \%)$ entre los mayores constituyentes de ingenieros, a las larvas de Coleoptera $(22,1 \%)$ y algunas familias de hemípteros $(11,8 \%)$ entre los herbívoros, y a determinadas familias de Araneae $(33,8 \%)$ y a Staphylinidae $(15,7 \%)$ entre los depredadores. También Pinzón et al. (2014) en bosques maduros de la Amazonia oriental brasileña concretan datos semejantes y añaden a Gastropoda y Diplopoda como principales táxones detritívoros y fundamentalmente a la familia Scarabaeidae de Coleoptera como taxon herbívoro.

En conclusión, los resultados obtenidos de la macrofauna del suelo en Vallecito con respecto a Helechal, principalmente la alta riqueza encontrada, están determinados en primera instancia por el distinto período estacional de muestreo en estos sitios, y también, por la retención de humedad en el suelo, como otro factor determinante. De modo general, los bosques siempreverdes en El Salón, Sierra del Rosario, tienen una alta riqueza taxonómica y abundancia de la macrofauna y una composición funcional dominada por ingenieros del suelo y detritívoros, estructura que es muy parecida a la de otros bosques del trópico húmedo. Esta estructura apenas cambia en otros sistemas de uso de la tierra estudiados en Cuba como en los bosques secundarios, pero sí en los pastizales y cultivos con una mayor perturbación y estrés sobre el suelo, donde la riqueza y abundancia disminuyen y dominan grupos ingenieros y herbívoros. En los últimos tiempos no se han publicado resultados de otros tipos de bosques en Cuba, pero la similitud de los resultados del presente estudio con los de otros bosques tropicales, sugiere que sería esperable una estructura de la comunidad semejante en otros bosques cubanos. No obstante, es necesario hacer estudios similares en otros tipos de bosques en Cuba para confirmar estos resultados o describir las particularidades de cada uno.

\section{AGRADECIMIENTOS}

A Jorge A. Sánchez Rendón del Instituto de Ecología y Sistemática de Cuba, por las sugerencias brindadas y arreglos realizados para mejorar el manuscrito. A los colegas Arturo Hernández, Yojana I. Menéndez, Jorge L. Guerra y Ormaily Madruga, por la ayuda ofrecida en la recolección de la macrofauna del suelo y por facilitar el acceso a las áreas de estudio. También a los tres revisores anónimos, por los cambios propuestos para lograr una adecuada presentación de los resultados.

\section{REFERENCIAS}

Amazonas NT, RAG Viani, MGA Rego, FF Camargo, RT Fujihara, OA Valsechi. 2018. Soil macrofauna density and diversity across a cronosequence of tropical forest restoration in Southeastern Brazil. Brazilian Journal of Biology 78(3): 449-456. DOI:10.1590/1519-6984.169014.

Anderson JM, JSI Ingram. 1993. Tropical soil biology and fertility. A handbook of methods. Wallingford, UK. CAB International. $221 \mathrm{p}$.

Brévault T, S Bikay, JM Maldes, K Naudin. 2007. Impact of a no-till with mulch soil management strategy on soil macrofauna communities in a cotton cropping system. Soil \& Tillage Research 97: 140-149.

Brown G, C Fragoso, I Barois, P Rojas, JC Patrón, J Bueno, A Moreno, P Lavelle, V Ordaz, C Rodríguez. 2001. Diversidad y rol funcional de la macrofauna edafica en los ecosistemas tropicales mexicanos. Acta Zoologica Mexicana 1: 79-110.

Cabrera Dávila G. 2017. Rectificaciones a la lista taxonómica de la macrofauna del suelo en bosques siempreverdes de la Sierra del Rosario, Artemisa, Cuba. Acta Botánica Cubana 216(3): 123-126.

Cabrera Dávila G, N Robaina, D Ponce de León. 2011a. Riqueza y abundancia de la macrofauna edáfica en cuatro usos de la tierra en las provincias de Artemisa y Mayabeque, Cuba. Pastos y Forrajes 34(3): 313-330.

Cabrera Dávila G, N Robaina, D Ponce de León. 2011b. Composición funcional de la macrofauna edáfica en cuatro usos de la tierra en las provincias de Artemisa y Mayabeque, Cuba. 
Pastos y Forrajes 34(3): 331-346.

De la Rosa N, S Negrete-Yankelevich. 2012. Distribución espacial de la macrofauna edáfica en bosque mesófilo, bosque secundario y pastizal en la reserva La Cortadura, Coatepec, Veracruz, México. Revista Mexicana de Biodiversidad 83: 201-215.

Driessen PO, J Deckers, C Spaar, F Nachtergeehe. 2001. Lecture notes on the mayor soil. FAO, Resources Report, Rome. $335 \mathrm{p}$.

Durán EH, JC Suárez. 2013. Fauna del suelo y hojarasca en arreglos agroforestales de la Amazonia Colombiana. Momentos de Ciencia 10(1): 59-66.

García Y, W Ramírez, S Sánchez. 2014. Efecto de diferentes usos de la tierra en la composición y la abundancia de la macrofauna edáfica en la provincia Matanzas. Pastos y Forrajes 37(3): 313-321.

Gómez DF, MC Godoy, JM Coronel. 2016. Macrofauna edáfica en ecosistemas naturales y agroecosistemas de la Ecoregión Esteros del Iberá (Corrientes, Argentina). Ciencia del Suelo 34(1): 43-56.

González R. 1984. Distribución vertical y dinámica de la abundancia y biomasa de Diplopoda en suelos de ecosistemas forestales de la Sierra del Rosario, Cuba. Poeyana 264: $1-27$.

González R. 1989. Estructura de las comunidades de Coleoptera de la hojarasca y del suelo de dos ecosistemas forestales de la Sierra del Rosario. Reporte de Investigación del Instituto de Ecología y Sistemática. Serie Zoológica 1: 3-14.

González R, A Herrera. 1983. La macrofauna del suelo del bosque siempreverde estacional de la Sierra del Rosario. (Resultados preliminares). Reporte de Investigación del Instituto de Zoología 10: 1-13.

Hernández A, JM Pérez, D Bosch, N Castro. 2015. Clasificación de los suelos de Cuba 2015. Mayabeque, Cuba. Ediciones INCA. 91 p.

Hernández L, JA Sánchez. 2012. Dinámica de la humedad del suelo y la fitomasa de raíces en ecosistemas de la Sierra del Rosario, Cuba. Pastos y Forrajes 35(1): 79-98.

Herrera RA, L Menéndez, ME Rodríguez, EE García. 1988. Ecología de los bosques siempreverdes de la Sierra del Rosario, Cuba. Montevideo, Uruguay. ROSTLAC. 760 p.

Martínez MA, JA Sánchez. 2000. Comunidades de lombrices de tierrra (Annelida: Oligochaeta) en un bosque siempre verde y un pastizal de Sierra del Rosario, Cuba. Caribbean Journal of Science 36: 94-103.

Marichal R, M Grimaldi, A Feijoo, J Oszwald, C Praxedes, D Hernan, M del Pilar Hurtado, T Desjardins, M Lopes, L Gonzaga, I Souza, M Nascimento, GG Brown, S Tsélouiko, M Bonifacio, T Decaëns, E Velasquez, P Lavelle. 2014. Soil macroinvertebrate communities and ecosystem services êêdeforested landscapes of Amazonia. Applied Soil Ecology 83: 177-185.

Pinzón S, GX Rousseau, A Rocha, D Celentano, ML Correa, H Braun. 2014. La macrofauna del suelo como indicadora de degradación de bosques ribereños en la amazonia oriental brasilera. Revista de la Facultad de Agronomía, La Plata 114(1): 49-60.

Rousseau L, SJ Fonte, O Téllez, R Van der Hoek, P Lavelle. 2013. Soil macrofauna as indicators of soil quality and land use impacts in smallholder agroecosystems of western Nicaragua. Ecological Indicators 27: 71-82.

Ruiz N, P Lavelle, J Jiménez. 2008. Soil Macrofauna Field Manual. Roma, Italy. Food and Agriculture Organization of the United Nations (FAO). 100 p.

Sánchez JA, BC Muñoz, L Montejo, M Lescaille, RA Herrera. 2012. Tamaño y nutrientes de semillas en 32 especies arbóreas de un bosque tropical siempreverde de Cuba y su relación con el establecimiento de las plántulas. Revista del Jardín Botánico Nacional 33: 181-204.

Souza ST, PC Cassol, D Baretta, MLC Bartz, O Klauberg, AL Mafra, M Gonçalves. 2016. Abundance and Diversity of Soil Macrofauna in Native Forest, Eucalyptus Plantations, Perennial Pasture, Integrated Crop-Livestock, and No-Tillage Cropping. Revista Brasileira de Ciência do Solo 40. DOI: $10.1590 / 18069657 \mathrm{rbcs} 20150248$.

Teixeira Y. 2007. Comparação da riqueza de Isoptera entre as florestas ombrófilas densas, mesófilas de altitude e matasde-cipó de altitude, no Domínio Atlântico do sul da Bahia. Tesis de Doctorado en Ciencias. Sao Paulo, Brasil. Facultad de Filosofía, Ciencias y Letras de Ribeirão Preto, Universidad de Sao Paulo. 97 p.

Zerbino MS, N Altier, A Morón, C Rodríguez. 2008. Evaluación de la macrofauna del suelo en sistemas de producción en siembra directa y con pastoreo. Agrociencia XII(1): 44-55. 
